\title{
An efficient analytic approach for solving Hiemenz flow through a porous medium of a non-Newtonian Rivlin-Ericksen fluid with heat transfer
}

https://doi.org/10.1515/nleng-2017-0160

Received December 5, 2017; revised December 31, 2017; accepted January 20, 2018.

\begin{abstract}
In the present work, the problem of Hiemenz flow through a porous medium of a incompressible nonNewtonian Rivlin-Ericksen fluid with heat transfer is presented and newly developed analytic method, namely the homotopy analysis method (HAM) is employed to compute an approximation to the solution of the system of nonlinear differential equations governing the problem. This flow impinges normal to a plane wall with heat transfer. It has been attempted to show capabilities and wide-range applications of the homotopy analysis method in comparison with the numerical method in solving this problem. Also the convergence of the obtained HAM solution is discussed explicitly. Our reports consist of the effect of the porosity of the medium and the characteristics of the Non-Newtonian fluid on both the flow and heat.
\end{abstract}

Keywords: Hiemenz Flow, Porous Medium, NonNewtonian Fluid, Heat Transfer, HAM

MSC: 34B15, 34B40

\section{Introduction}

The two dimensional flow of a fluid near a stagnation point was first examined by Hiemenz who demonstrated

\footnotetext{
Kourosh Parand, Department of Computer Sciences, Faculty of Mathematical Sciences, Shahid Beheshti University, G.C. Tehran, Iran

and Department of Cognitive Modeling, Institute for Cognitive and Brain Sciences, Shahid Beheshti University, G.C. Tehran, Iran, E-mail: k_parand@sbu.ac.ir

Yasaman Lotfi, Department of Computer Sciences, Faculty of Mathematical Sciences, Shahid Beheshti University, G.C. Tehran, Iran, E-mail: yasaman.yasi94@gmail.com

*Corresponding Author: Jamal Amani Rad, Department of Cognitive Modeling, Institute for Cognitive and Brain Sciences, Shahid Beheshti University, G.C. Tehran, Iran, E-mail: j.amanirad@gmail.com;j_amanirad@sbu.ac.ir
}

that the Navier-Stokes equations governing the flow can be reduced to an ordinary differential equation of third order using similarity transformation [1]. In order to include various physical effect, stagnation point flow was extended in numerous ways. It is of great technical importance, for example in the prediction of skin-friction as well as heat/mass transfer near stagnation regions of bodies in high speed flows and also in the design of thrust bearings and radial diffusers, drag reduction, transpiration cooling and thermal oil recovery. The axisymmetric three dimensional stagnation point flow was studied by Homann [2]. In hydromagnetics, the problem of Hiemenz flow was chosen by Na to illustrate the solution of a thirdorder boundary value problem using the technique of finite differences. Ariel [3] gave approximate solution of the same problem. Attia [4] in the presence of uniform suction or injection investigated the effect of an externally applied uniform magnetic field on the two or three-dimensional stagnation point flow. One of the important subject with many engineering applications is the study of heat transfer in boundary layer flows. Massoudi and Ramezan [5] used a perturbation technique to solve for the stagnation point flow and heat transfer of a Non-Newtonian fluid of second grade. Their result is valid only for small values of the parameter that determines the behavior of the Non-Newtonian fluid. The problem was extended to nonisothermal surface by Massoudi and Ramezan. Garg [6] improved the solution obtained by Massoudi by computing numerically the flow characteristics for any value of the Non-Newtonian parameter using a pseudo-similarity solution.

In recent years, there has been some interest on flows of Non-Newtonian fluids where inertial effects are significant. Boundary layer theories for fluid similar to a second grade fluid have been formulated by Rajeswari and Rathna, Bhatnagar, Beard and Waiters, and Frater. Rajagopal et al. developed a boundary layer approximation for a second grade fluid [7].

Because of the equations of motion of a second grade fluid are one order higher than the Navier-Stokes equations, we need a boundary condition in addition to the 
usual adherence boundary condition. Beard and Waiters [8] used a perturbation procedure, using the coefficient that multiplies the highest order term in the equation as the perturbation parameter thereby lowering the order of the equation.

Due to the widespread applications such as oil recovery, paper and textile coating, composite manufacturing processes, mixture theory, filtration processes, geothermal engineering, insulation systems, flow through porous media received substantial attention. The attempts to include porous media in the flows of the complex fluids need some new physical parameters besides Non-Newtonian fluid parameters. Thus, Darcys equations or some generalization of it depending on pressure field, not neglecting porosity, are appropriate to study this type of flows thorough the porous media which is rigid or nearly rigid solid. Liu [9] examined flow of an electrically conducting fluid of second grade thorough porous media subject to transverse magnetic field over the stretching sheet with power-law surface temperature.

Channel flow with porous medium of a MHD-driven second-grade fluid have been considered by Hayat and Abbas [10]. MHD flow of a Sisko Fluid occupying porous media have been investigated by Khan et al. [11]. Yih [12] has reported on the effects of uniform suction or blowing and magnetic field on the heat transfer characteristics of the Hiemenz problem in porous media.

Simultaneous heat transfer from different geometrics embedded in porous media has many engineering and geophysical applications such as geothermal reservoirs, drying of porous solids, thermal insulation, enhanced oil recovery, packed-bed catalytic reactors, cooling of nuclear reactors, and underground energy transport. Nakayama and Koyama [13] investigated free convection over a vertical flat plate embedded in a thermally stratified porous medium. Gorla et al. [14] have considered the effects of thermal dispersion and stratification on mixed convection about a vertical surface in a porous medium.

The heat transfer characteristics of mixed convection flow over a vertical flat plate embedded in porous medium is investigated by Hassanien et al. [15].

The influence of variable viscosity on forced convection heat transfer over a flat plate in a porous medium is examined by Seddeek. Also, Seddeek [16] studied the thermal radiation and buoyancy effects on MHD free convection heat generation flow over an accelerating permeable surface with temperature dependent viscosity.
Modeling of natural or physical phenomena leads to solving nonlinear differential equations (DEs) arise in bounded or unbounded domains. Solution of these equations is not easy especially through analytical and numerical approach. Therefore, endless efforts of researchers are directed to find ways to solve them or to decrease the error in solutions [17-20]. In 1992, Liao [21] employed the basic ideas of the homotopy in topology to propose a general analytic method for nonlinear problems, namely homotopy analysis method (HAM), [22-26]. Based on homotopy of topology, the validity of the HAM is independent of whether or not there exist small parameters in the considered equation. Therefore, the HAM can overcome the foregoing restrictions and limitations of perturbation methods [27]. Furthermore, the HAM always provides us with a family of solution expressions in the auxiliary parameter $\hbar$. The convergence region and rate of each solution might be determined conveniently by the auxiliary parameter $\hbar$. The HAM also avoids discretization and provides an efficient numerical solution with high accuracy, minimal calculation and avoidance of physically unrealistic assumptions. Besides, the HAM is rather general and contains the homotopy perturbation method (HPM) [11-12], the Adomian decomposition method (ADM) [28] and $\delta$-expansion method. In fact, HPM and ADM are always special cases of HAM when $\hbar=-1$. The convergence of HAM solution series is dependent upon three factors, that is, the initial guess, the auxiliary linear operator, and the auxiliary parameter $\hbar$. However, as a special case of homotopy analysis method, the convergence of HPM solution series is only dependent upon two factors: the auxiliary linear operator and the initial guess. So, given the initial guess and the auxiliary linear operator, HPM cannot provide other ways to ensure that the solution is convergent. When the HPM is applied to a problem, a good enough initial approximation and/or auxiliary operator are necessary to ensure that the solution is convergent. However, as pointed out by Liao [22], there are no rigorous theories to direct us to choose the initial approximations, auxiliary linear operators, auxiliary functions, and auxiliary parameter $\hbar$. From the practical viewpoints, there are some fundamental rules such as the rule of solution expression, the rule of coefficient ergodicity, and the rule of solution existence, which play important roles within the HAM. Unfortunately, the rule of solution expression implies such an assumption that we should have, more or less, some knowledge about a given non-linear problem a prior. So, theoretically, this assumption impairs the HAM, although we can always attempt some base functions even if a given non-linear problem is completely new for us. 
In recent years, the homotopy analysis method has been successfully employed to solve many types of nonlinear problems such as the nonlinear equations arising in heat transfer [29], the nonlinear model of diffusion and reaction in porous catalysts [30], the chaotic dynamical systems [31], the non-homogeneous Blasius problem [32], the generalized three-dimensional MHD flow over a porous stretching sheet [33], the wire coating analysis using MHD Oldroyd 8-constant fluid [34], the axisymmetric flow and heat transfer of a second grade fluid past a stretching sheet [35], the MHD flow of a second grade fluid in a porous channel [36], the generalized Couette flow [37], the squeezing flow between two infinite plates [38], the steady three-dimensional problem of condensation film on inclined rotating disk [39], the Burger and regularized long wave equations [40], the laminar viscous flow in a semi-porous channel in the presence of a uniform magnetic field [41], the flow and diffusion of chemically reactive species over a nonlinearly stretching sheet immersed in a porous medium [42], the heat transfer over an unsteady stretching permeable surface with prescribed wall temperature [43], the unsteady three-dimensional MHD flow and mass transfer in a porous space [44], the unsteady MHD flow of Maxwellian fluids above impulsively stretching sheets [45], the developed MHD micropolar fluid flow between vertical porous plates [46], the Sturm-Liouville problems [47], the gas flow through a micro-nano porous media [48], the Poiseuille flow of a third grade fluid in a porous medium [49], the heat transfer in pipe flow of a Johnson-Segalman fluid [50], the SIR (SusceptiblesInfectives-Removed) models of epidemics [51], the generalized Zakharov equation [52], the fractional Lorenz system [53] and other problems [54-66]. All of these successful applications verified the validity, effectiveness and flexibility of the HAM.

This paper is arranged as follows: in Section 2, a mathematical model has been proposed to describe the non-newtonian behavior of Rivlin-Ericksen fluid with heat transfer. In Section 3, we apply our method to the problem. In Section 4, in order to achieve proper $\hbar$ we minimize the Euclidean norm of residual. In the last section, we present our results through tables and figures to show how accurate is our results. Also, a comparison is made between the analytical results with those of previously reported along with the numerical results obtained by radial basis functions method [67, 68]. Finally, the conclusions are described in this section.

\section{Problem formulation}

There are many fluids whose behavior cannot be described by the classical Navier-Stokes model. One of the many models that has been proposed to describe the nonnewtonian behavior of such fluid is the incompressible fluid of second grade. In present study, these fluid impinges perpendicular to a plane directed along $x$-axis. A plane potential flow arriving from $y$-axis, impinges on the flat plate situated at $y=0$ and divides in two streams and leaves the wall in two directions. The flow near the wall must satisfy the wall boundary conditions. Far away the wall, we reach the invisid potential flow sliding along the walls. The component of velocity in the cartesian coordinates $(x, y)$ has been defined as $u$ and $v$. But $U$ and $V$ are the potential velocity component . The velocity distribution in the frictionless region in the neighborhood of the stagnation point is given by

$$
\begin{gathered}
U(x)=c x, \\
V(y)=-c y,
\end{gathered}
$$

where $c>0$ is the dimensional constant which is proportional to far field velocity. In these fluids the Cauchy stress $T$ is related to the fluid motion in the following manner:

$$
T=-p I+\mu A_{1}+\alpha_{1} A_{2}+\alpha_{2} A_{1}^{2},
$$

where $p$ denotes the pressure, $I$ is the identity tensor, $\mu$ is the viscosity of the fluid, the scalar constant $\alpha_{1}$ and $\alpha_{2}$ are the normal stress moduli , $A_{1}$ and $A_{2}$ are the first twoRevlin-Ericksen tensors given by:

$$
\begin{aligned}
& A_{1}=\nabla \vec{V}+(\nabla \vec{V})^{T}, \\
& A_{2}=\frac{D A_{1}}{D t}+A_{1} \nabla \vec{V}+(\nabla \vec{V})^{T} A_{1},
\end{aligned}
$$

where $\frac{D}{D t}$ is the material time derivative, so with respect to Eq. (2.2) we have:

$$
\frac{D A_{1}}{D t}=\frac{\partial A_{1}}{\partial t}+\left(\nabla A_{1}\right) \vec{V} .
$$

The newtonian fluid is described when $\alpha_{1}=\alpha_{2}=0$. Here we consider the case of the reduced Revlin-Ericksen fluid in which $\alpha_{2}=0$. Mass conservation is written as:

$$
\nabla V=0 .
$$

We use momentum equation which is non newtonian porous medium analog of the Navier-Stokes equation. We neglect body forces such as gravity. Darcy's law revealing a proportionality between flow rate and the applied pressure difference is used in momentum equation. The momentum equation is:

$$
\rho \frac{d V}{d t}=\nabla T+R,
$$


where $T$ is cauchy stress and the darcy resistance $(R)$ is:

$$
R=\frac{-\mu u}{k_{p}},
$$

where the coefficient $k_{p}$ is independent of the nature of the fluid but it depends on the geometry of the medium. It has dimensions (length) ${ }^{2}$ and is called permeability. Then, by using the usual boundary layer approximations and stress component and porosity force the continuity and momentum equations Eqs. (2.3) and (2.4) for two-dimensional steady-state flows reduce to:

$$
\begin{aligned}
& \frac{\partial u}{\partial x}+\frac{\partial v}{\partial y}=0, \\
& \rho\left(u \frac{\partial u}{\partial x}+v \frac{\partial u}{\partial y}\right)=U \frac{d U}{d x}+\mu \frac{\partial^{2} u}{\partial y^{2}}-\frac{\mu u}{k_{p}} \\
& \quad+\alpha_{1}\left(\frac{\partial u}{\partial y} \frac{\partial^{2} u}{\partial y^{2}}+v \frac{\partial^{3} u}{\partial y^{3}}+\frac{\partial\left(u \frac{\partial^{2} u}{\partial y^{2}}\right)}{\partial x}\right) .
\end{aligned}
$$

The energy equation for temperature $T$ by neglecting viscous dissipation terms and applying boundary layer approximations, is given by:

$$
\rho c_{p}\left(u \frac{\partial T}{\partial x}+v \frac{\partial T}{\partial y}\right)=k \frac{\partial^{2} T}{\partial y^{2}},
$$

where $T$ is the fluid temperature, $c_{p}$ is specific heat capacity at constant pressure and $k$ is the fluid thermal conductivity.

The boundary conditions on wall and far from it is written as:

$$
\begin{gathered}
y=0 \Rightarrow\left\{\begin{array}{l}
u=0 \\
v=0 \\
T=T_{w}
\end{array}\right. \\
y \rightarrow \infty \Rightarrow\left\{\begin{array}{l}
u \rightarrow c x \\
\frac{\partial u}{\partial y} \rightarrow 0 \\
T \rightarrow T_{\infty}
\end{array}\right.
\end{gathered}
$$

where $T_{\infty}$ is the constant temperature of the fluid far away from the wall and $T_{w}$ is the wall temperature. Next applying similarity relations:

$$
\begin{aligned}
& u(x, y)=c x f^{\prime}(\eta), \\
& v(x, y)=-\sqrt{c v} f(\eta), \\
& \eta=\sqrt{c / v} y,
\end{aligned}
$$

where the prime denotes differentiation with respect to $\eta$. $v$ is kinematic viscosity and defined as $v=\mu / \rho$. We apply the non-dimensional variable for heat transfer equation as follow:

$$
\theta=\frac{T-T_{\infty}}{T_{w}-T_{\infty}} .
$$

By using the similarity relations Eqs. (2.10) and (2.11), continuity relation , Eq. (2.5) is identically satisfied and it can be shown that similarity solution of the Eq. (2.6) exists for the stagnation point flow for which we get:

$$
\begin{aligned}
& K\left(f f^{(4)}-2 f^{\prime} f^{\prime \prime \prime}+f^{\prime \prime 2}\right)-f^{\prime \prime \prime}-f f^{\prime \prime}+f^{\prime 2}-M\left(1-f^{\prime}\right)-1=0, \\
& \theta^{\prime \prime}+P r f \theta^{\prime}=0 .
\end{aligned}
$$

By applying similarity variables Eqs. (2.10) and (2.11), the new transformated boundary conditions are written as:

$$
\begin{aligned}
& f(0)=f^{\prime}(0)=0, f^{\prime}(\infty)=1, f^{\prime \prime}(\infty)=0, \\
& \theta(0)=1, \theta(\infty)=0,
\end{aligned}
$$

where $K$ is dimensionless normal stress modulus, $K=$ $\alpha_{1} c / \mu, M$ is the porosity parameter, $M=v / c k_{p}$, and $P r=$ $\mu c_{p} / k$ is prandtl number. The flow equation is constitutes non-linear, non-homogeneous boundary value problem. By computing $T$ in terms of $\theta$, we have :

$$
T=\theta\left(T_{w}-T_{\infty}\right)+T_{\infty},
$$

by differentiating Eq. (2.15) relative to $\eta$, it has been written as:

$$
\frac{\partial T}{\partial y}=\frac{\partial \theta}{\partial \eta} \sqrt{c / v}\left(T_{w}-T_{\infty}\right),
$$

the heat transfer at the wall with respect to Fourier's law and Eq. (2.16) is given by:

$$
q_{w}=-\left.k \frac{\partial T}{\partial y}\right|_{y=0}=-k \theta^{\prime}(0) \sqrt{c / v}\left(T_{w}-T_{\infty}\right) .
$$

Thus, $\theta^{\prime}(0)$ is proportional to wall heat transfer.

For boundary layer flow, the wall skin friction tensor $\tau_{w}$ is given by:

$$
\tau_{w}=\left.\mu \frac{\partial u}{\partial y}\right|_{y=0},
$$

where $\mu$ is the viscosity coefficient. By use of Eq. (2.18), the skin friction coefficient $c_{f}$ can be defined as:

$$
c_{f}=\frac{\tau_{w}}{\rho U^{2}} .
$$

By investigating Eq. (2.10), the differentiation of $u$ relative to $y$ on the wall is given by:

$$
\begin{aligned}
& \frac{\partial u}{\partial y}=U f^{\prime \prime}(\eta) \frac{\partial \eta}{\partial y}, \\
& \left.\frac{\partial u}{\partial y}\right|_{y=0}=\sqrt{c / v} U f^{\prime \prime}(0) .
\end{aligned}
$$


While substituting the Eqs. (2.18) and (2.19) in it , the new form of Eq. (2.20) is written as:

$$
\frac{c_{f} U}{v \sqrt{c / v}}=f^{\prime \prime}(0) \text {. }
$$

By applying Eq. (2.1) in above equation, we have:

$$
c_{f} \sqrt{\frac{U x}{v}}=f^{\prime \prime}(0) .
$$

Subsequently, $f^{\prime \prime}(0)$ by use of Reynolds's number is defined as:

$$
c_{f} R e^{1 / 2}=f^{\prime \prime}(0),
$$

where $R e=U x / v$.

With respect to Eq. (2.21), the variable $f^{\prime \prime}(0)$ is proportional to skin friction coefficient.

Thus, because of their relation to physical quantities, we discuss the $\theta^{\prime}(0)$ and $f^{\prime \prime}(0)$ in our results.

\section{Method of solution}

The first step in the HAM is to find a set of base functions to express the sought solution of the problem under investigation. As mentioned by Liao [22], a solution may be expressed with different base functions, among which some converge to the exact solution of the problem faster than others. Such base functions are obviously better suited for the final solution to be expressed in terms of. Noting these facts, we have decided to express $f(\eta)$ and $\theta(\eta)$ by a set of base functions of the form

$$
\left\{\eta^{q} \exp (-m \eta), q \geq 0, m \geq 0\right\},
$$

and write

$$
\begin{aligned}
& f(\eta)=\sum_{m=1}^{+\infty} \sum_{q=0}^{\infty} a_{q, m} \eta^{q} e^{-m \eta}, \\
& \theta(\eta)=\sum_{m=1}^{+\infty} \sum_{q=0}^{\infty} b_{q, m} \eta^{q} e^{-m \eta},
\end{aligned}
$$

where $a_{q, m}$, and $b_{q, m}$ are the coefficients to be determined later using the governing equations, Eqs. (2.12) and (2.13), and its corresponding boundary conditions Eq. (2.14). This expression (3.2) and (3.3) satisfies the rule of solution expression for $f(\eta)$ and $\theta(\eta)$ as required in the HAM [22]. According to this rule, and with an eye on the boundary conditions as given by Eq. (2.14), we choose an initial guess for the solution in the following form:

$$
\begin{aligned}
& f_{0}(\eta)=\eta-1+e^{-\eta}, \\
& \theta_{0}(\eta)=e^{-\eta} .
\end{aligned}
$$

In the second step, we need to choose an appropriate auxiliary linear operator. The basic idea of the HAM is to transform the nonlinear governing equation into an infinite number of linear equations with the advantage that unlike other techniques, one has the freedom to choose the linear operator. Moreover, in the HAM it is not necessary for the linear operator to be of the same order as the original nonlinear equation (for more details see Ref. [69]). Using these ideas, we introduce the auxiliary linear operators in the form of

$$
\begin{aligned}
& \mathcal{L}_{f}[\phi(\eta ; p)]=\left(\frac{\partial^{4}}{\partial \eta^{4}}+\frac{\partial^{3}}{\partial \eta^{3}}\right) \phi(\eta ; p), \\
& \mathcal{L}_{\theta}[\psi(\eta ; p)]=\left(\frac{\partial^{2}}{\partial \eta^{2}}+\frac{\partial}{\partial \eta}\right) \psi(\eta ; p),
\end{aligned}
$$

with the properties

$\mathcal{L}_{f}\left[C_{1}+C_{2} \eta+C_{3} \eta^{2}+C_{4} e^{-\eta}\right]=0, \quad \mathcal{L}_{\theta}\left[D_{1}+D_{2} e^{-\eta}\right]=0$, where $\left\{C_{i}\right\}_{i=1}^{4}$ and $\left\{D_{i}\right\}_{i=1}^{2}$ are integral constants to be determined by boundary conditions. Obviously, $C_{3}$ should be chosen to be zero because we seek a finite solution. From Eqs. (2.12) and (2.13) we define nonlinear operators

$$
\begin{gathered}
\mathcal{N}_{f}[\phi(\eta ; p), \psi(\eta ; p)]=K\left(\phi \frac{\partial^{4} \phi}{\partial \eta^{4}}-2 \frac{\partial \phi}{\partial \eta} \frac{\partial^{3} \phi}{\partial \eta^{3}}+\left(\frac{\partial^{2} \phi}{\partial \eta^{2}}\right)^{2}\right) \\
-\frac{\partial^{3} \phi}{\partial \eta^{3}}-\phi \frac{\partial^{2} \phi}{\partial \eta^{2}}+\left(\frac{\partial \phi}{\partial \eta}\right)^{2}-M\left(1-\frac{\partial \phi}{\partial \eta}\right)-1, \\
\mathcal{N}_{\theta}[\phi(\eta ; p), \psi(\eta ; p)]=\frac{\partial^{2} \phi}{\partial \eta^{2}}+\operatorname{Pr} \phi \frac{\partial \psi}{\partial \eta},
\end{gathered}
$$

and then construct the homotopy

$$
\begin{aligned}
& \mathcal{H}_{f}[\phi(\eta ; p), \psi(\eta ; p)]=(1-p) \mathcal{L}_{f}\left[\phi(\eta ; p)-f_{0}(\eta)\right] \\
& -\hbar p H_{1}(\eta) \mathcal{N}_{f}[\phi(\eta ; p), \psi(\eta ; p)], \\
& \mathcal{H}_{\theta}[\phi(\eta ; p), \psi(\eta ; p)]=(1-p) \mathcal{L}_{\theta}\left[\psi(\eta ; p)-\theta_{0}(\eta)\right] \\
& -\hbar p H_{2}(\eta) \mathcal{N}_{\theta}[\phi(\eta ; p), \psi(\eta ; p)],
\end{aligned}
$$

where $\hbar \neq 0$ is the convergence-control parameter, $H_{1}(\eta)$ and $H_{2}(\eta)$ are auxiliary functions. Setting $\mathcal{H}_{i}[\phi(\eta ; p), \psi(\eta ; p)]=0$, for $i=f, \theta$, we have the zeroorder deformation problems as follows:

$(1-p) \mathcal{L}_{f}\left[\phi(\eta ; p)-f_{0}(\eta)\right]=\hbar p H_{1}(\eta) \mathcal{N}_{f}[\phi(\eta ; p), \psi(\eta ; p)]$,

$(1-p) \mathcal{L}_{\theta}\left[\psi(\eta ; p)-\theta_{0}(\eta)\right]=\hbar p H_{2}(\eta) \mathcal{N}_{\theta}[\phi(\eta ; p), \psi(\eta ; p)]$,

The boundary conditions for this equations are

$$
\begin{aligned}
& \phi(0 ; p)=0,\left.\quad \frac{\partial \phi(\eta ; p)}{\partial \eta}\right|_{\eta=0}=0,\left.\quad \frac{\partial \phi(\eta ; p)}{\partial \eta}\right|_{\eta=\infty}=1, \\
& \left.\frac{\partial^{2} \phi(\eta ; p)}{\partial \eta^{2}}\right|_{\eta=\infty}=0, \\
& \psi(0 ; p)=1, \quad \psi(\infty ; p)=0,
\end{aligned}
$$


where $p \in[0,1]$ is an embedding parameter. Clearly, when $p=0$, the solutions to Eqs. (3.7) and (3.8) are given by

$$
\phi(\eta ; 0)=f_{0}(\eta), \quad \psi(\eta ; 0)=\theta_{0}(\eta),
$$

and when $p=1$, because of $\hbar \neq 0$ and $\left\{H_{i}(\eta)\right\}_{i=1}^{2} \neq 0$ the solutions are equivalent to those of Eqs. (2.12) and (2.13) provided that the conditions

$$
\phi(\eta ; 1)=f(\eta), \quad \psi(\eta ; 1)=\theta(\eta),
$$

are satisfied. Therefore, as the embedding parameter $p$ increases from 0 to $1, \phi(\eta ; p)$ varies continuously from the initial guess solutions $f_{0}(\eta)$ to the exact solutions $f(\eta)$ of Eq. (2.12), and so $\psi(\eta ; p)$ from the initial guess solution $\theta_{0}(\eta)$ to the exact solutions $\theta(\eta)$ of Eq. (2.13). The process is the so-called deformation in topology. With the aid of terms used in topology, Eqs. (3.7) and (3.8) are correspondingly called zero-order deformation equations of HAM, and detailed descriptions can be found in Ref. [22]. Therefore, $f(\eta ; p)$ and $\theta(\eta ; p)$ can be expanded in a Taylor series with respect to $p$ to provide

$$
\begin{aligned}
& \phi(\eta ; p)=f_{0}(\eta)+\sum_{m=1}^{+\infty} f_{m}(\eta) p^{m}, \\
& \psi(\eta ; p)=\theta_{0}(\eta)+\sum_{m=1}^{+\infty} \theta_{m}(\eta) p^{m},
\end{aligned}
$$

where

$$
f_{m}(\eta)=\left.\frac{1}{m !} \frac{\partial^{m} \phi(\eta ; p)}{\partial p^{m}}\right|_{p=0}, \theta_{m}(\eta)=\left.\frac{1}{m !} \frac{\partial^{m} \psi(\eta ; p)}{\partial p^{m}}\right|_{p=0} .
$$

Obviously, the convergence region of the series (3.11) and (3.12) depends upon the auxiliary linear operators $\mathcal{L}_{f}$ and $\mathcal{L}_{\theta}$, the auxiliary parameter $\hbar$, and the two non-zero auxiliary functions $H_{1}(\eta)$ and $H_{2}(\eta)$. If all of them are properly chosen so that the convergences of the above two series at $p=1$ are guaranteed, the series solutions of Eqs. (2.12) and (2.13) can be expressed as follows

$$
\begin{aligned}
& f(\eta)=f_{0}(\eta)+\sum_{m=1}^{+\infty} f_{m}(\eta), \\
& \theta(\eta)=\theta_{0}(\eta)+\sum_{m=1}^{+\infty} \theta_{m}(\eta) .
\end{aligned}
$$

To make the mathematics more tractable, we define the vectors

$$
\begin{aligned}
\vec{f}_{m} & =\left\{f_{0}, f_{1}, \ldots, f_{m}\right\}, \\
\vec{\theta}_{m} & =\left\{\theta_{0}, \theta_{1}, \ldots, \theta_{m}\right\} .
\end{aligned}
$$

At this stage in the analysis $f_{m}(\eta)$ and $\theta_{m}(\eta)$ are still unknown. Now, in order to obtain $f_{m}(\eta)$ and $\theta_{m}(\eta)$, we first differentiate the zero-order deformation equations $m$ times with respect to $p$, divide the equation so obtained by $m$ ! and finally set $p=0$. With these maneuvering, the so-called high-order deformation equation is obtained for $f_{m}(\eta)$ and $\theta_{m}(\eta)$ as

$$
\begin{aligned}
& \mathcal{L}_{f}\left[f_{m}(\eta)-\chi_{m} f_{m-1}(\eta)\right]=\hbar H_{1}(\eta) \Re_{1, m}\left(\vec{f}_{m-1}\right), \\
& \quad(m=1,2,3, \ldots), \\
& \mathcal{L}_{\theta}\left[\theta_{m}(\eta)-\chi_{m} \theta_{m-1}(\eta)\right]=\hbar H_{2}(\eta) \Re_{2, m}\left(\vec{\theta}_{m-1}\right), \\
& \quad(m=1,2,3, \ldots),
\end{aligned}
$$

where we have

$$
\begin{gathered}
\Re_{1, m}\left(\vec{f}_{m-1}\right)=\left.\frac{1}{(m-1) !} \frac{\partial^{m-1} \mathcal{N}_{f}[\phi(\eta ; p), \psi(\eta ; p)]}{\partial p^{m-1}}\right|_{p=0}, \\
\Re_{2, m}\left(\vec{\theta}_{m-1}\right)=\left.\frac{1}{(m-1) !} \frac{\partial^{m-1} \mathcal{N}_{\theta}[\phi(\eta ; p), \psi(\eta ; p)]}{\partial p^{m-1}}\right|_{p=0} ^{(3.19)},
\end{gathered}
$$

and

$$
\chi_{m}= \begin{cases}0, & \text { if } m \leq 1, \\ 1, & \text { if } m>1 .\end{cases}
$$

It should be noted that $\Re_{1, m}\left(\vec{f}_{m-1}\right)$ and $\Re_{2, m}\left(\vec{\theta}_{m-1}\right)$ only depends on $\vec{f}_{m-1}$ and $\vec{\theta}_{m-1}$ and is defined as

$$
\begin{aligned}
& \Re_{1, m}\left(\vec{f}_{m-1}\right)=K\left[\sum_{i=0}^{m-1} f_{i} f_{m-i-1}^{(4)}-2 \sum_{i=0}^{m-1} f_{i}^{\prime} f_{m-i-1}^{(3)}+\sum_{i=0}^{m-1} f_{i}^{\prime \prime} f_{m-i-1}^{\prime \prime}\right] \\
& -f_{m-1}^{\prime \prime \prime}-\sum_{i=0}^{m-1} f_{i} f_{m-i-1}^{\prime \prime}+\sum_{i=0}^{m-1} f_{i}^{\prime} f_{m-i-1}^{\prime}+M f_{m-1}^{\prime} \\
& -(M+1)\left(1-\chi_{m}\right),
\end{aligned}
$$

and

$$
\mathfrak{R}_{2, m}\left(\vec{\theta}_{m-1}\right)=\theta_{m-1}^{\prime \prime}+\operatorname{Pr} \sum_{i=0}^{m-1} f_{i} \theta_{m-i-1}^{\prime} .
$$

The boundary conditions for Eqs. (3.17) and (3.18) are

$$
\begin{aligned}
& f_{m}(0)=0, f_{m}^{\prime}(0)=0, f_{m}^{\prime}(\infty)=0, f_{m}^{\prime \prime}(\infty)=0 \\
& \theta_{m}(\eta)=0, \theta_{m}(\infty)=0,
\end{aligned}
$$

The general solution of Eqs. (3.17) and (3.18) are

$$
\begin{aligned}
& f_{m}(\eta)=\hat{f}_{m}(\eta)+C_{1}+C_{2} \eta+C_{3} \eta^{2}+C_{4} e^{-\eta}, \\
& \theta_{m}(\eta)=\hat{\theta}_{m}(\eta)+D_{1}+D_{2} e^{-\eta},
\end{aligned}
$$

where $\left\{C_{i}\right\}_{i=1}^{4}$ and $\left\{D_{i}\right\}_{i=1}^{2}$ are constants, $\hat{f}_{m}(\eta)$ and $\hat{\theta}_{m}(\eta)$ are special solutions of Eqs. (3.17) and (3.18), respectively. 
According to the rule of solution expression denoted by Eqs. (3.2) and (3.3), $C_{3}=0$. The other unknown are governed by

$$
\begin{aligned}
& \hat{f}_{m}(0)+C_{1}+C_{4}=0, \hat{f}_{m}^{\prime}(0)+C_{2}-C_{4}=0, \\
& \hat{f}_{m}^{\prime}(\infty)+C_{2}=0, \quad \hat{\theta}_{m}(0)+D_{1}+D_{2}=0, \\
& \hat{\theta}_{m}(\infty)+D_{1}=0 .
\end{aligned}
$$

and according to our algorithm, the another boundary conditions are satisfied. In this way, it is easy to obtain the terms $f_{m}(\eta)$ and $\theta_{m}(\eta)$ for $m=1,2,3, \ldots$, of Eqs. (3.17) and (3.18) one after the other (say, using symbolic softwares such as Mathematica or Maple).

In order to satisfy the rule of solution expression, the rule of coefficient ergodicity, and the rule of solution existence $[22,70]$, we have some freedom in choosing the auxiliary function $H(\eta)$. It is found that converged solutions can be obtained using either $H(\eta)=1$ or $H(\eta)=e^{-\kappa \eta}$ where $\kappa$ is an integer. In practice, however, it was realized that using the latter results in a series which converges faster. Therefore, in the following sections we use $H_{1}(\eta)=H_{2}(\eta)=e^{-\eta}$. At the $N$ th-order approximation, we have below form of analytical solution of Eqs. (2.12) and (2.13)

$$
f(\eta) \approx F_{N}(\eta)=\sum_{i=0}^{N} f_{i}(\eta), \quad \theta(\eta) \approx \Theta_{N}(\eta)=\sum_{i=0}^{N} \theta_{i}(\eta) .
$$

$N$ must be sufficiently large. In practice, we decided to st the calculations at $N=15$ (15-order) having realized tr. a sufficiently small tolerance has been met.

Thus, the nonlinear Eqs. (2.12) and (2.13) are converted ir a series of linear boundary value problems as in Eqs. (3.: and (3.18), which can be easily solved by symbolic comp tation software such as Mathematica and Maple.

\section{Convergence of HAM solution}

HAM provides us with great freedom in choosing the lution of a non-linear problem by different base functior This has a great effect on the convergence region because the convergence region and rate of a series are chiefly determined by the base functions used to express the solution. Therefore, we can approximate a non-linear problem more efficiently by choosing a proper set of base functions and ensure its convergency. On the other hand, as pointed out by Liao in his book [22], the convergence and rate of approximation for the HAM solution strongly depends on the value of auxiliary parameter $\hbar$. Even if the initial approximation, the auxiliary linear operator, and the auxiliary function are given, we still have great freedom to choose the value of the auxiliary parameter $\hbar$. So, the auxiliary parameter $\hbar$ provides us with an additional way to conveniently adjust and control the convergence region and rate of solution series. By means of the so-called $\hbar$-curves it is easy to find out the so-called valid regions of $\hbar$ to gain a convergent solution series. When the valid region of $\hbar$ is a horizontal line segment then the solution is converged. It is worthwhile to be mentioned that for different values of parameters $(K, M, P r)$ a new $\hbar$-curve should be plotted. As using a unique $\hbar$-curve for all cases may lead to a considerable error. Therefore, in this study we have obtained admissible values of $\hbar$ for all cases; for example in our case study according to Figure 1 we depicted the $\hbar$-curve of $f^{\prime \prime}(0)$ and $\theta^{\prime}(0)$ for $K=1, M=0, P r=0.5$. By considering the $\hbar$-curve we can obtain the reasonable interval for $\hbar$ in each case. Figures 2-3 show how auxiliary parameters varied with changing $K$ and $M$. If $K$ increases then the range of convergency of solution is restricted and then decreased. According to Figures 3 by increasing $M$, the range of convergency is decreased. Also, according to Figures 4 the acceptable range of auxiliary parameter $\hbar$ of $f^{\prime \prime}(0)$ and $\theta^{\prime}(0)$ for $K=0.5, M=0, P r=0.5$ is $-1.3<\hbar<0$ and $-1.4<\hbar<0$, respectively.

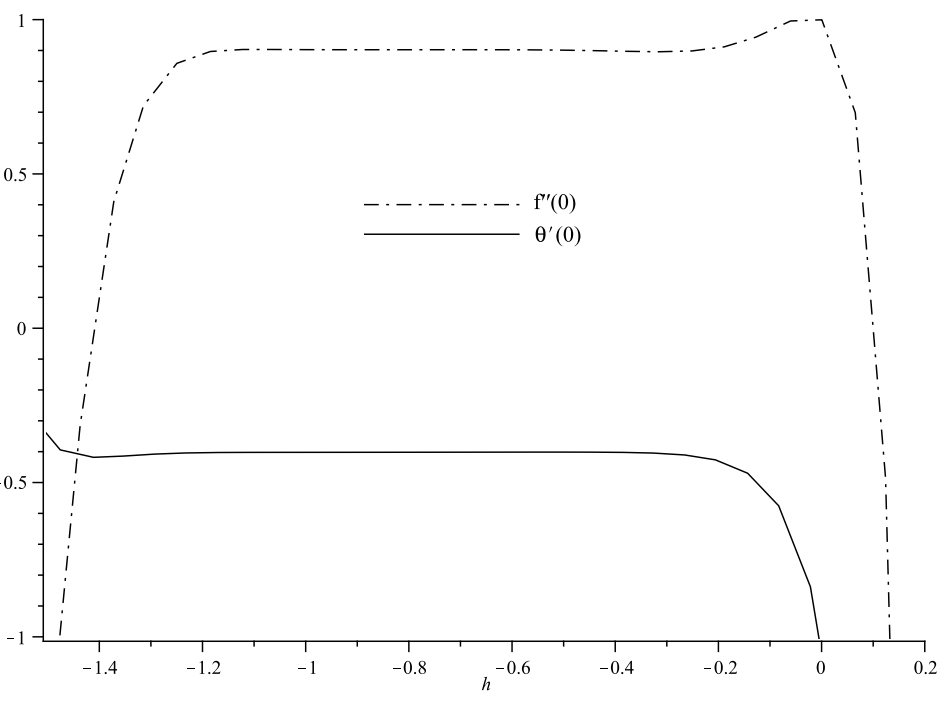

Fig. 1: The $\hbar$-validity curve obtained by the 15 th-orther approximation of the HAM for various functions when $K=0.5, M=0$ and $\operatorname{Pr}=0.5$.

To choose optimal value of auxiliary parameter, the averaged residual errors (see Ref. [71] for more details) of 


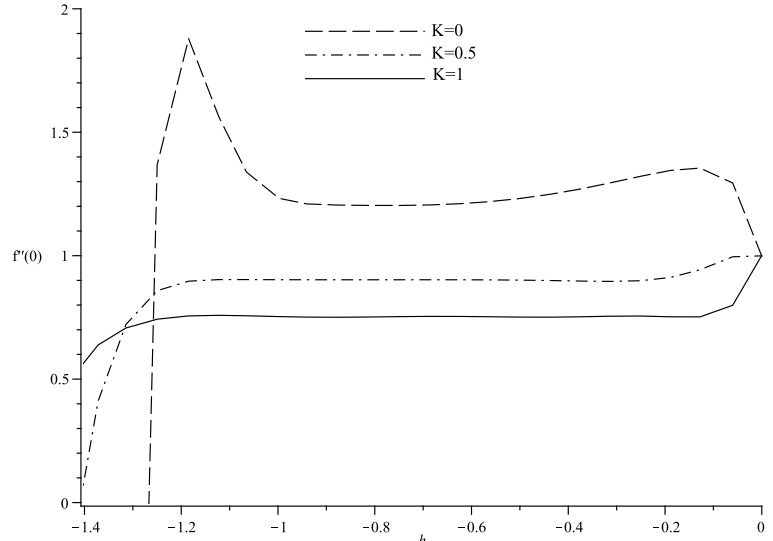

(a) The $\hbar$-curves of $f^{\prime \prime}(0)$

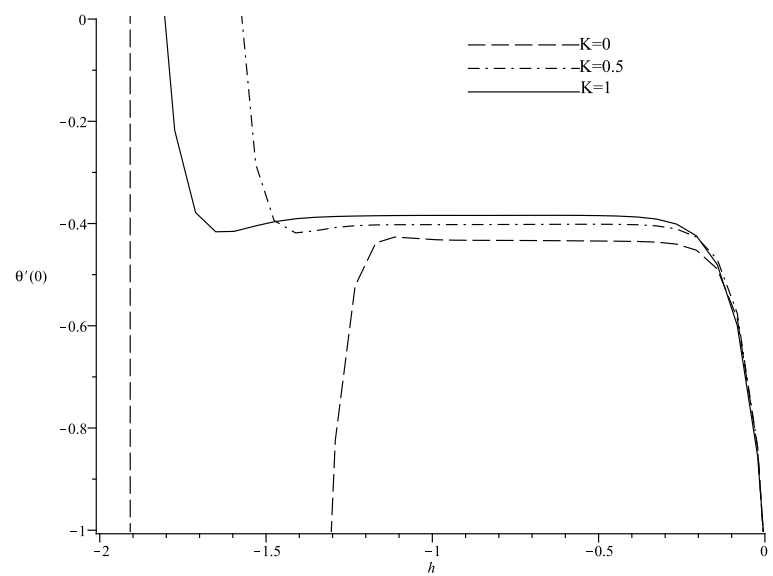

(b) The $\hbar$-curves of $\theta^{\prime}(0)$

Fig. 2: The $\hbar$-curves of $f^{\prime \prime}(0)$ and $\theta^{\prime}(0)$ obtained by the 15 th-orther approximation of the HAM for different values $K$, when $M=0$ and $\operatorname{Pr}=0.5$.

Eq. (2.12) and Eq. (2.13) are defined as

$$
\begin{aligned}
& R_{1}(\eta, \hbar)=K\left(F_{N} F_{N}^{(4)}-2 F_{N}^{\prime} F_{N}^{(3)}+\left(F_{N}^{\prime \prime}\right)^{2}\right)-F_{N}^{(3)} \\
& \quad-F_{N} F_{N}^{\prime \prime}+\left(F_{N}^{\prime}\right)^{2}-M\left(1-F_{N}^{\prime}\right)-1, \\
& R_{2}(\eta, \hbar)=\Theta_{N}^{\prime \prime}+\operatorname{Pr}_{N} \Theta_{N}^{\prime} .
\end{aligned}
$$

We try to minimize $\left\|R_{j}(\eta, \hbar)\right\|^{2}$ (for $j=1,2$ ) by choosing good auxiliary parameter $\hbar$. We define $\left\|R_{j}(\eta, \hbar)\right\|^{2}$ as

$r_{j, N}=\left\|R_{j}(\eta, \hbar)\right\|^{2}=\int_{0}^{b} R_{j}^{2}(\eta, \hbar) d \eta \simeq \sum_{j=0}^{n} \omega_{j} R_{j}^{2}\left(\frac{b}{2} s_{j}+\frac{b}{2}, \hbar\right)$

$$
j=1,2,
$$

where

$$
\begin{aligned}
& \omega_{j}=\frac{b}{\left(1-s_{j}^{2}\right)\left(\left.\frac{d}{d s} P_{n+1}(s)\right|_{s=s_{j}}\right)^{2}}, j=0,1, \ldots, n, \\
& P_{n+1}\left(s_{j}\right)=0, j=0,1, \ldots, n,
\end{aligned}
$$

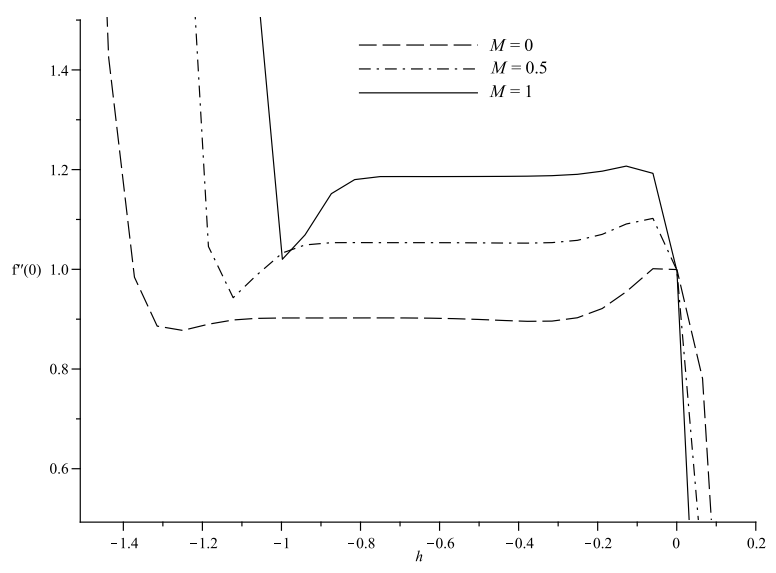

(a) The $\hbar$-curves of $f^{\prime \prime}(0)$

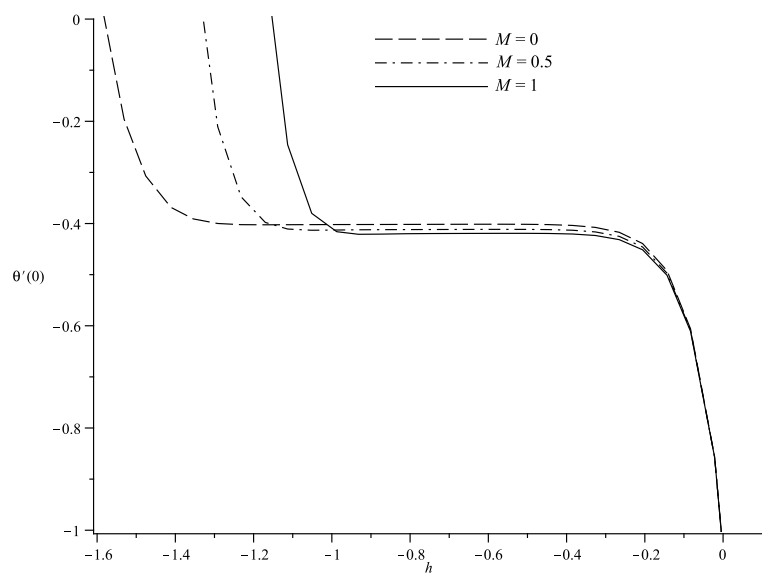

(b) The $\hbar$-curves of $\theta^{\prime}(0)$

Fig. 3: The $\hbar$-curves of $f^{\prime \prime}(0)$ and $\theta^{\prime}(0)$ obtained by the 15 th-orther approximation of the HAM for different values $M$, when $K=0.5$ and $\operatorname{Pr}=0.5$.

$P_{n+1}(s)$ is $(n+1)$ th-order Legendre polynomial and $b$ is the big value that satisfies $\int_{b}^{\infty} R_{j}^{2}\left(\eta, \hbar_{j}\right) d \eta<\varepsilon_{\hbar}$ and we take $b=7$ and $n=50$. The optimal values of $\hbar$ are given by the minimum of $r_{j, N}$ on interval which Figures 6 and 8 are shown as

$$
\frac{\partial r_{j, N}}{\partial \hbar}=0, \quad j=1,2 .
$$

By solving two above equations, the optimal values of $\hbar$ for $f(\eta)$ and $\hbar$ for $\theta(\eta)$ are obtained and the series solutions lead to appropriate convergence rate. we call the optimal value of $\hbar$ for $f(\eta)$ and $\hbar$ for $\theta(\eta)$ by name $\hbar_{1}$ and $\hbar_{2}$, respectively. The values of residual norm for some $N$ and appropriate parameters $\hbar_{1}$ and $\hbar_{2}$ are reported in Table 1. Also, to see the accuracy of the solutions, the residual errors are shown for 15th- order approximation and deferent value of parameters in Figures 5 and 7. 


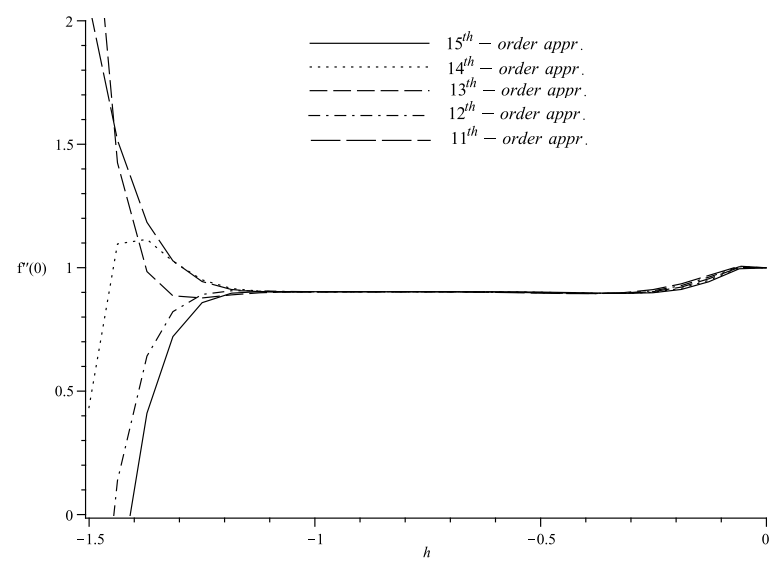

(a) The $\hbar$-curves of $f^{\prime \prime}(0)$

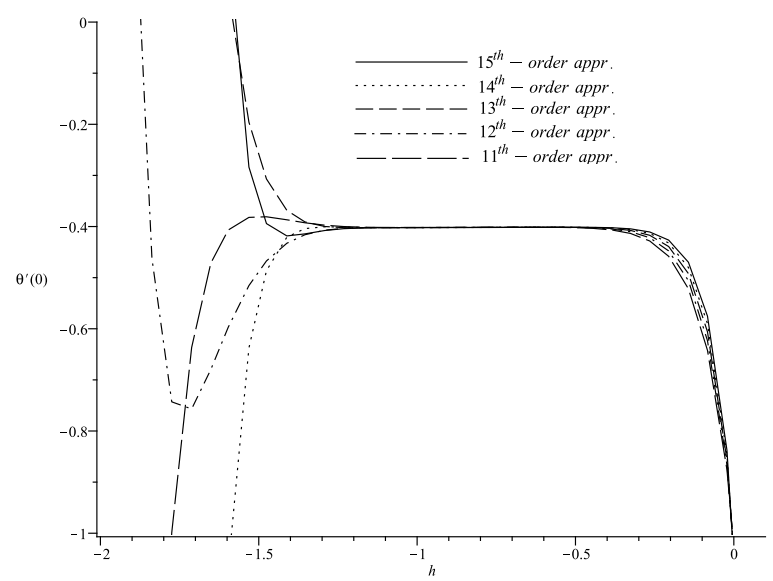

(b) The $\hbar$-curves of $\theta^{\prime}(0)$

Fig. 4: The $\hbar$-curves of $f^{\prime \prime}(0)$ and $\theta^{\prime}(0)$ obtained by deferent order approximation of the HAM for values $M=0, K=0.5$ and $P r=0.5$.

Table 1: The values of residual norm for some $N$ and appropriate parameters $\hbar_{1}$ and $\hbar_{2}$ for case of $M=0, K=0.5$ and $P r=0.5$.

\begin{tabular}{lcl|cl}
\hline $\boldsymbol{N}$ & $\boldsymbol{r}_{\mathbf{1}}\left(\hbar_{\mathbf{1}}\right)$ & $\hbar_{\mathbf{1}}$ & $\boldsymbol{r}_{\mathbf{2}}\left(\hbar_{\mathbf{2}}\right)$ & $\hbar_{\mathbf{2}}$ \\
\hline 5 & $4.4070 e-4$ & -0.54 & $2.6787 e-4$ & -1.26 \\
6 & $2.2486 e-6$ & -1.00 & $1.2787 e-4$ & -1.22 \\
7 & $8.1677 e-7$ & -0.92 & $7.8698 e-5$ & -1.19 \\
8 & $8.0255 e-7$ & -0.91 & $3.7723 e-5$ & -1.22 \\
9 & $2.9701 e-7$ & -0.87 & $2.5174 e-5$ & -1.21 \\
10 & $1.5122 e-7$ & -0.81 & $1.8647 e-5$ & -1.16 \\
11 & $8.3853 e-8$ & -0.78 & $1.0244 e-5$ & -1.17 \\
12 & $5.2537 e-8$ & -0.73 & $5.9614 e-6$ & -1.19 \\
13 & $3.9879 e-8$ & -0.71 & $4.6492 e-6$ & -1.16 \\
14 & $3.3774 e-8$ & -0.68 & $2.9863 e-6$ & -1.14 \\
15 & $3.1812 e-8$ & -0.66 & $1.7164 e-6$ & -1.16 \\
\hline
\end{tabular}

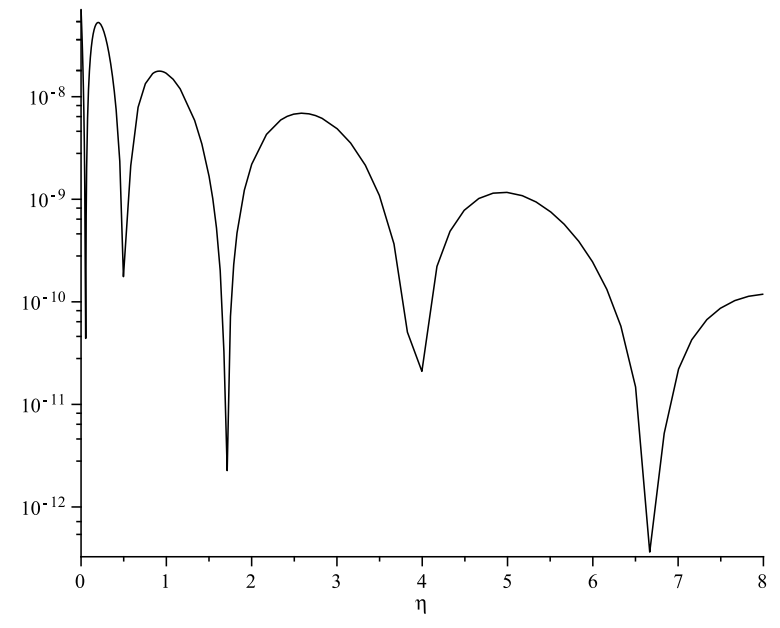

(a) The logarithmic square residual error for Eq. (2.12)

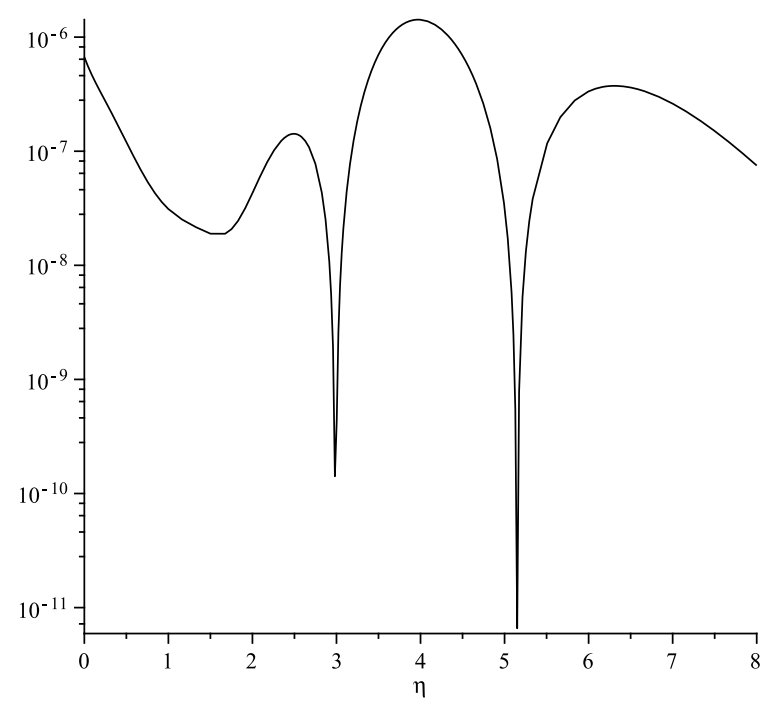

(b) The logarithmic square residual error for Eq. (2.13)

Fig. 5: The logarithmic square residual error for Eqs. (2.12) and (2.13) using 15th-order of approximations when $\hbar_{1}=-0.66$ and $\hbar_{2}=-1.16$ (optimal value), $K=0.5, M=0$ and $P r=0.5$.

\section{Concluding remarks}

\subsection{Results}

In this section, the numerical results of the model equations presented in Section 1, obtained by using HAM are discussed. For this proposed and have a clear insight of the problem, we displayed the numerical results with the help of graphical illustrations and tables.

Figures 9(a) and 10(a) illustrate the influence of the dimensionless normal stress $K$ on the velocity velocity boundary layer thickness $f^{\prime}(\eta)$, when the porosity parameter $M$ and prandtl number $\operatorname{Pr}$ are $M=0, P r=0.5$ and 


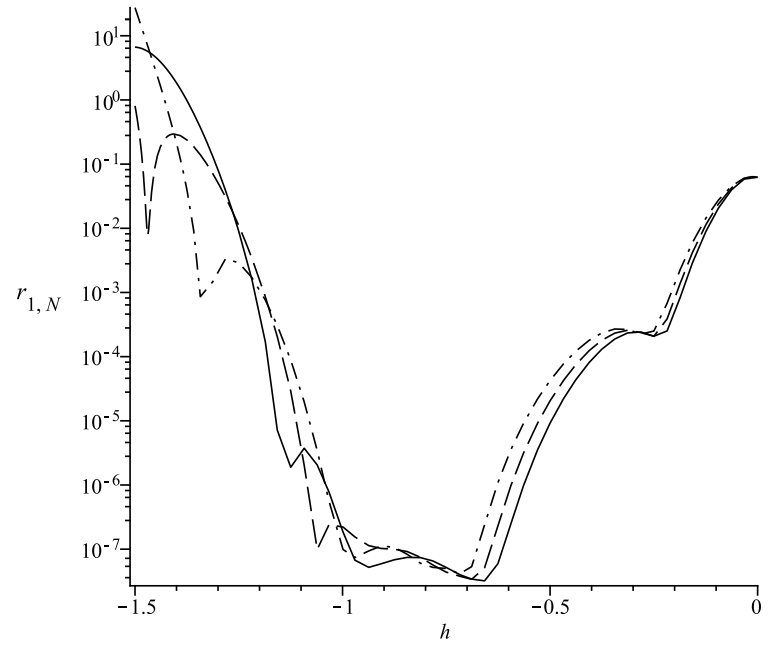

(a) The residual error $r_{1, N}$

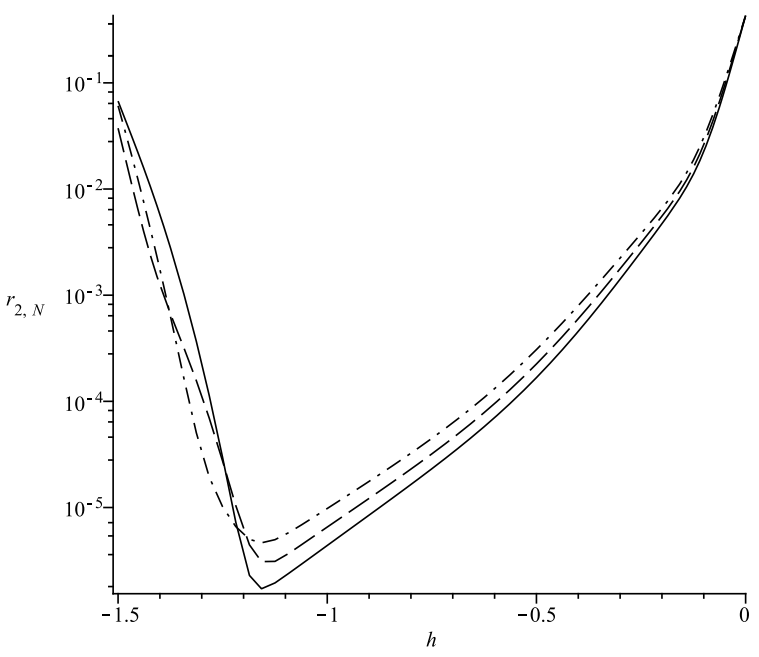

(b) The residual error $r_{2, N}$

Fig. 6: The residual error $r_{1, N}$ and $r_{2, N}$ versus $\hbar$ in case of $K=0.5$, $M=0$ and $P r=0.5$. Solid line: 15th-order approximation; Dashed line: 14 th-order approximation; Dash-dotted line: 13 th-order approximation.

$M=0.5, P r=0.5$, respectively. Figure 9(a) shows that $f^{\prime}(\eta)$ decrease when the dimensionless normal stress $K$ increases.

In Figures 9(b) and 10(b), we illustrates the temperature profile $\theta(\eta)$ for the different values of the porosity parameter $M$, where it can be seen that $\theta(\eta)$ increase when the porosity parameter $M$ increases.

Figures 11(a) and 11(b) show the effect of $M$ as a porosity parameter of the fluid on the $f^{\prime}(\eta)$ and $\theta(\eta)$ as the velocity profile and the temperature profile, respectively. It is observed that by increasing the value of $M$, the velocity profile $f^{\prime}(\eta)$ increases. Also, the parameter $M$ have the

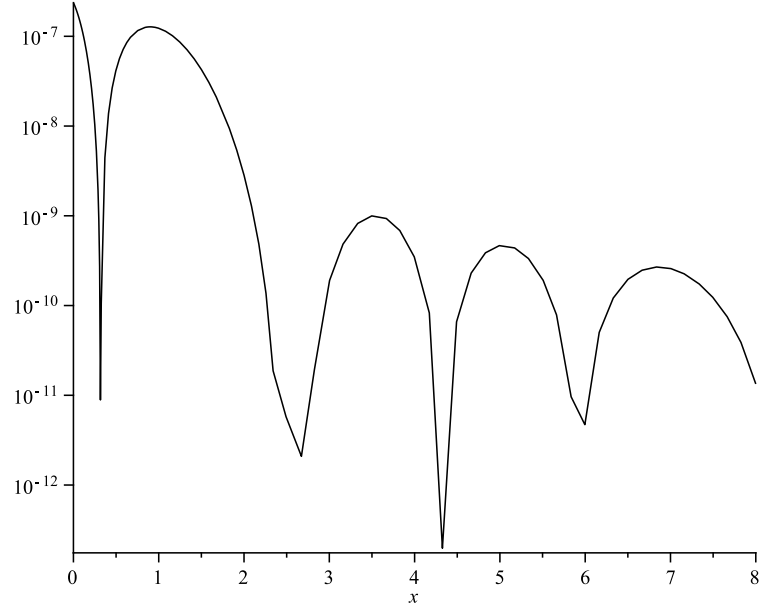

(a) The logarithmic square residual error for Eq. (2.12)

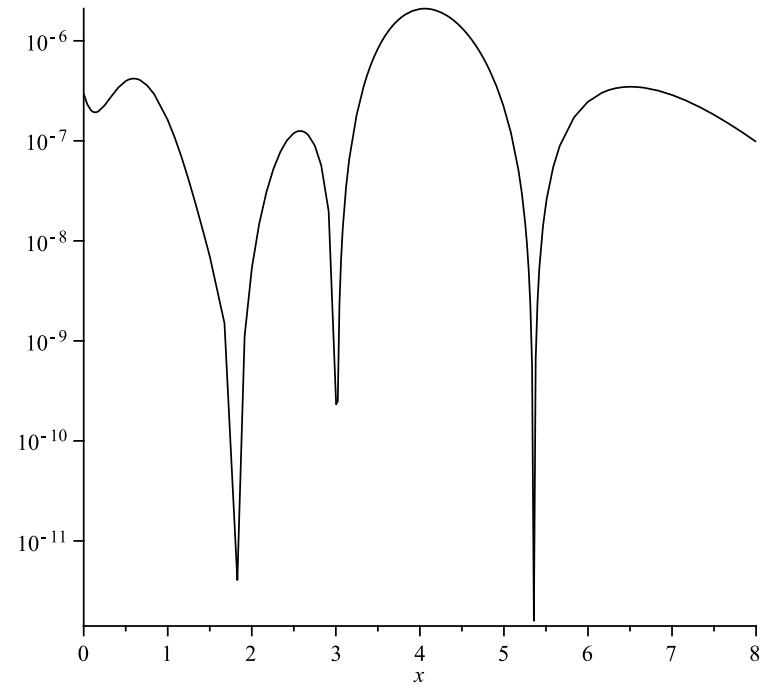

(b) The logarithmic square residual error for Eq. (2.13)

Fig. 7: The logarithmic square residual error for Eqs. (2.12) and (2.13) using 15th-order of approximations when $\hbar_{1}=-0.86$ and $\hbar_{2}=-1.29$ (optimal value), $K=1, M=0.5$ and $P r=0.5$.

few effect on the temperature profile $\theta(\eta)$, in order to with increase of the parameter $M$ the temperature profile $\theta(\eta)$ decrease.

Table 2 shows variations of the wall shear stress $f^{\prime \prime}(0)$ relative to $K$ and $M(P r=0.5)$ for 15th- order approximation. In constant porosity parameter $(M)$, the variable wall shear stress decreases as $K$ increases. Also, the wall shear stress increases steadily with the increment in $M$ for all values of $K$. Thus, as porosity parameter $(M)$ increases, We encounter the skin friction coefficient increment.

Also, Table 3 shows variations of the wall heat transfer rate $\theta^{\prime}(0)$ relative to $K$ and $M(P r=0.5)$ for 15th- order approximation. In constant porosity parameter $(M)$, the variable 


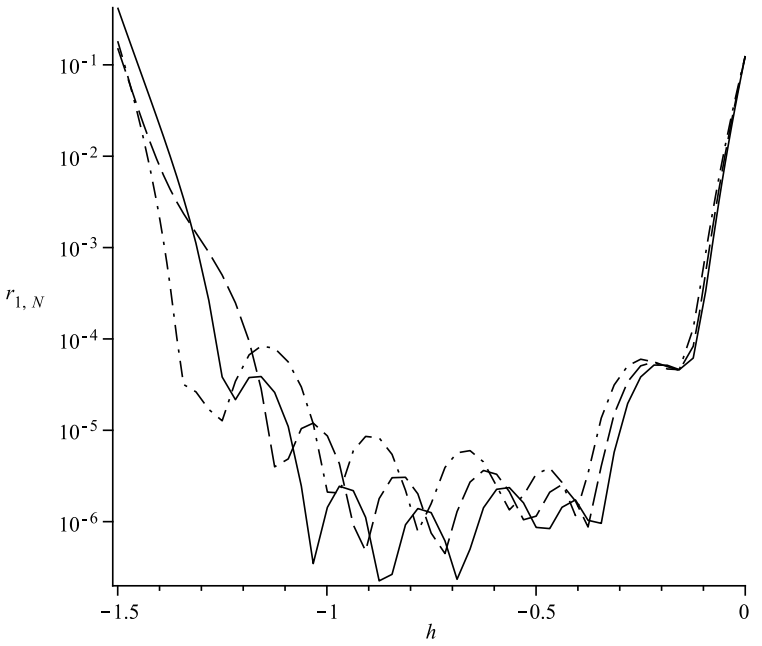

(a) The residual error $r_{1, N}$

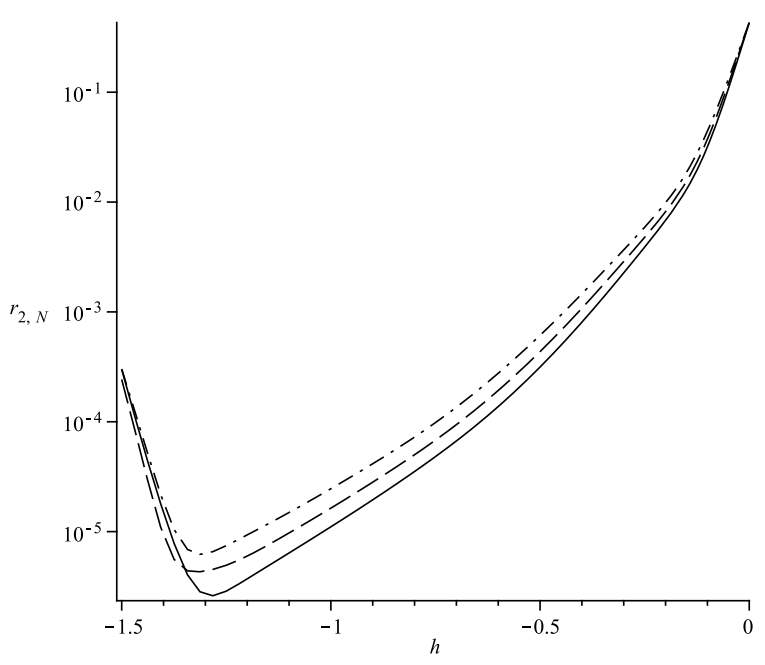

(b) The residual error $r_{2, N}$

Fig. 8: The residual error $r_{1, N}$ and $r_{2, N}$ versus $\hbar$ in case of $K=1$, $M=0.5$ and $P r=0.5$. Solid line: 15 th-order approximation; Dashed line: 14th-order approximation; Dash-dotted line: 13thorder approximation.

wall heat transfer rate decreases as $K$ increase. The wall heat transfer rate increases steadily with the increment in $M$ for all values of $K$.

In these tables, our results are compared with those of Attia [72]. Further, the Gaussian radial basis function method [29] with shape parameter $c=1$ and iteration number $N=20$ is also utilized as a criteria to verify our results. During the study the reliability and effectiveness of the applied method are demonstrated.

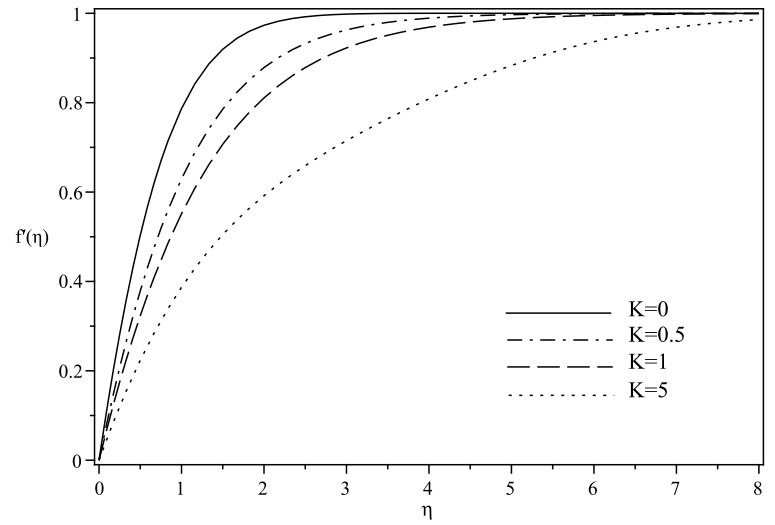

(a) The graph of $f^{\prime}(\eta)$

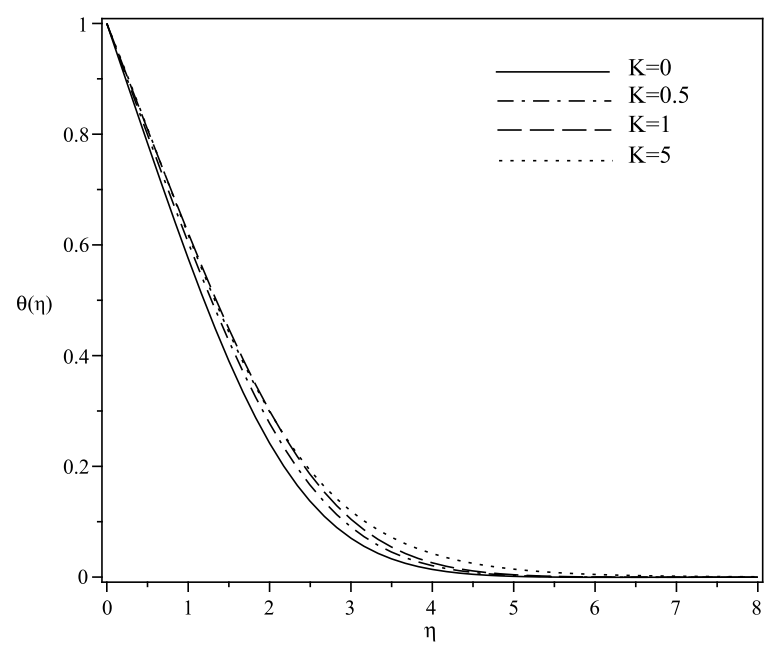

(b) The graph of $\theta(\eta)$

Fig. 9: The graphs of $f^{\prime}(\eta)$ and $\theta(\eta)$ for $M=0, P r=0.5$ and different values of $K$.

\subsection{Conclusion}

The two-dimensional stagnation point flow through a porous medium of a viscous incompressible nonNewtonian Rivlin-Ericksen fluid with heat transfer is studied. A analytical solution for the governing equations was obtained that allows the computation of the flow and heat transfer characteristics for various values of the non-Newtonian parameter $K$, the porosity parameter $M$, and the Prandtl number Pr. The results indicate that increasing the parameter $K$ increases the thermal boundary layer thickness and decreases the velocity profile while the porosity of the medium has a reversed effect. Besides, the homotopy analysis method provides us with freedom to choose the auxiliary parameter $\hbar$ and the auxiliary function $H(\eta)$. Also, with $\hbar$-curves we can control the speed of convergence. The solutions showed the validity and 
Table 2: Variation of the wall shear stress $f^{\prime \prime}(0)$ with $P r=0.5$.

\begin{tabular}{|c|c|c|c|c|c|c|c|c|c|c|c|c|}
\hline \multirow[b]{3}{*}{$M$} & \multicolumn{3}{|c|}{$K=0$} & \multicolumn{3}{|c|}{$\mathrm{K}=0.5$} & \multicolumn{3}{|c|}{$K=1$} & \multicolumn{3}{|c|}{$K=5$} \\
\hline & \multicolumn{2}{|c|}{ Present methods } & \multirow[b]{2}{*}{ Attia[72] } & \multicolumn{2}{|c|}{ Present methods } & \multirow[b]{2}{*}{ Attia[72] } & \multicolumn{2}{|c|}{ Present methods } & \multirow[b]{2}{*}{ Attia[72] } & \multicolumn{2}{|c|}{ Present methods } & \multirow[b]{2}{*}{ Attia[72] } \\
\hline & HAM & Numerical & & HAM & Numerical & & HAM & Numerical & & HAM & Numerical & \\
\hline 0 & 1.2326 & 1.2326 & 1.2326 & 0.9025 & 0.9026 & 0.9025 & 0.7528 & 0.7529 & 0.7528 & 0.4130 & 0.4130 & 0.4130 \\
\hline 0.5 & 1.4156 & 1.4150 & 1.3283 & 1.0536 & 1.0536 & 0.9810 & 0.8849 & 0.8849 & 0.8215 & 0.4938 & 0.4945 & 0.4555 \\
\hline 1 & 1.5846 & 1.5844 & 1.5840 & 1.1860 & 1.1860 & 1.1860 & 1.0000 & 1.0002 & 1.0000 & 0.5646 & 0.5648 & 0.5641 \\
\hline
\end{tabular}

Table 3: Variation of the wall shear stress $-\theta^{\prime}(0)$ with $\operatorname{Pr}=0.5$.

\begin{tabular}{lllllllll}
\hline & \multicolumn{2}{c}{$\mathrm{K}=0$} & \multicolumn{2}{c}{$\mathrm{K}=0.5$} & \multicolumn{2}{c}{$\mathrm{K}=1$} & & \\
\hline$M$ & HAM & Numerical & HAM & Numerical & HAM & Numerical & HAM & Numerical \\
\hline 0 & 0.4383 & 0.4383 & 0.4039 & 0.4039 & 0.3833 & 0.3833 & 0.3297 & 0.3297 \\
0.5 & 0.4502 & 0.4502 & 0.4109 & 0.4109 & 0.3944 & 0.3944 & 0.3414 & 0.3414 \\
1 & 0.4597 & 0.4597 & 0.4195 & 0.4195 & 0.4037 & 0.4037 & 0.3517 & 0.3517 \\
\hline
\end{tabular}

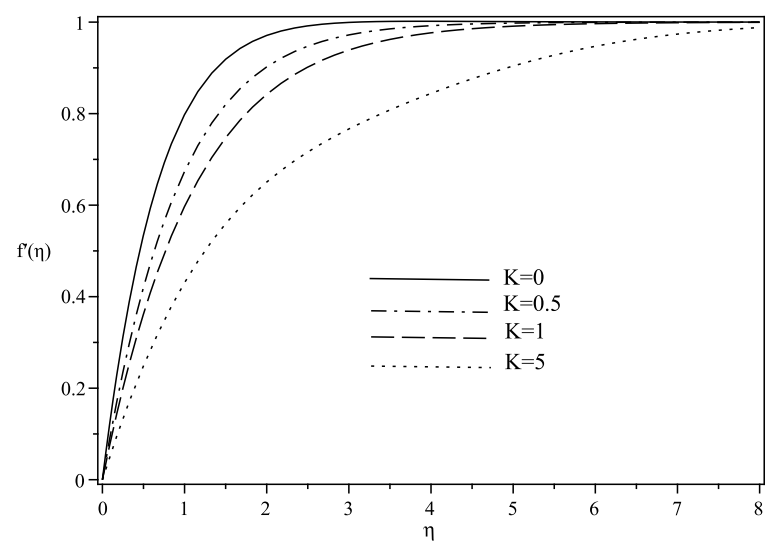

(a) The graph of $f^{\prime}(\eta)$

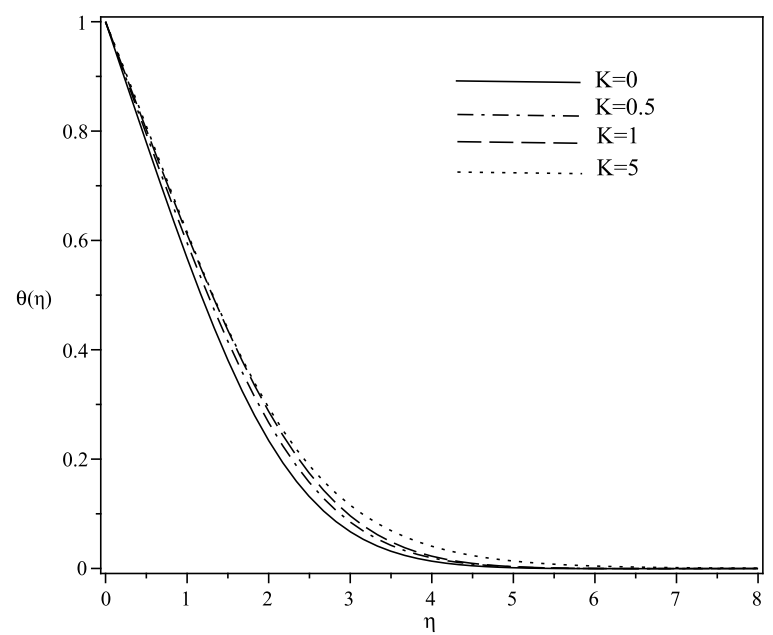

(b) The graph of $\theta(\eta)$

Fig. 10: The graphs of $f^{\prime}(\eta)$ and $\theta(\eta)$ for $M=0.5, \operatorname{Pr}=0.5$ and different values of $K$.

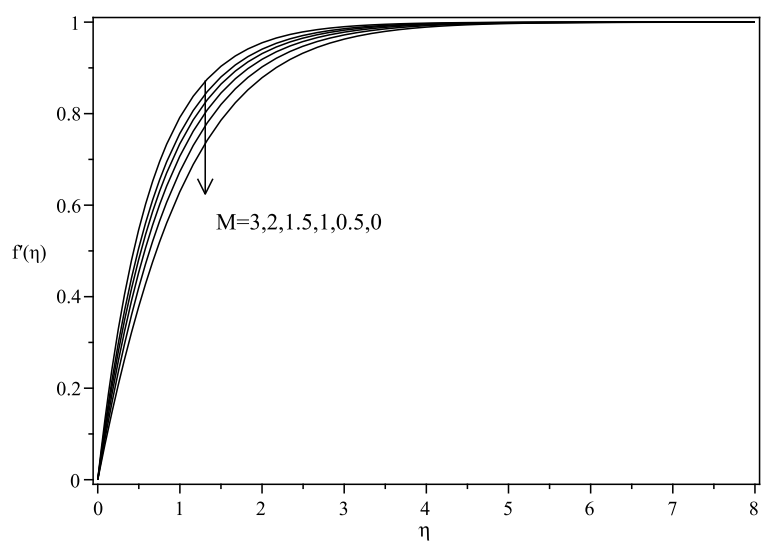

(a) The graph of $f^{\prime}(\eta)$

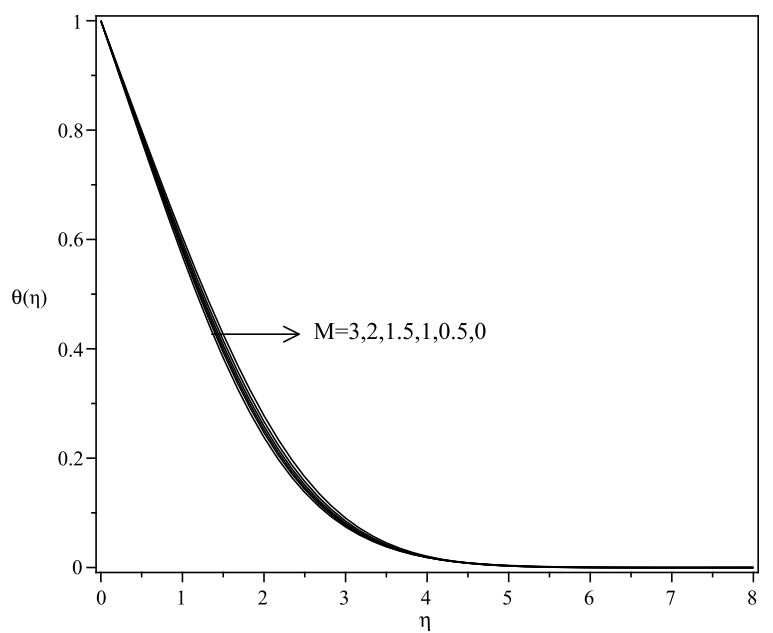

(b) The graph of $\theta(\eta)$

Fig. 11: The graphs of $f^{\prime}(\eta)$ and $\theta(\eta)$ for $K=0.5, \operatorname{Pr}=0.5$ and different values of $M$. 
potential of the homotopy analysis method for nonlinear problems in the science and engineering. Last but not least of all is the notion that the analytical approach drawn in this work is of a general nature meaning that it can easily be extended to other steady flow problems dealing with Newtonian and/or non-Newtonian fluids.

\section{References}

[1] K. Hiemenz, Die Grenzschicht an einem in den gleich formigen flussig keitsstrom eingetacuhten geraden krebzylinder, Dingl. Polytech. J. 32 (1911) 321-324.

[2] F. Homann, Der Einfluss grosser Zähigkeit bei der Strömung um den Zylinder und um die Kugel, Z. Angew. Math. Mech. (ZAMM) 16 (1936) 153-164.

[3] P. D. Ariel, Hiemenz flow in hydromagnetics, Acta Mech. 103 (1994) 31-43.

[4] H. A. Attia, Hydromagnetic stagnation point flow with heat transfer over a permeable surface, Arab. J. Sci. Eng. 28 (2003) 107-112.

[5] M. Massoudi, M. Ramezan, Boundary Layers Heat Transfer Analysis of a Viscoelastic Fluid at a Stagnation Point, ASME HTD 130 (1990) 81-86.

[6] V. K. Garg, Heat transfer Due to stagnation point flow of a nonNewtonian fluid, Acta. Mech. 104 (1994) 159-171.

[7] V. K. Garg, K. R. Rajagopal, Stagnation point flow of a nonNewtonian fluid, Mech. Res. Comm. 17 (1990) 415-421.

[8] D. W. Beard, K. Waiters, Stagnation point flow of a nonNewtonian fluid, Proc. Camb. Phil. Soc. 60 (1964) 667-674.

[9] I. Liu, Flow and heat transfer of an electrically conducting fluid of second grade in porous medium over a stretching sheet subject to a transverse magnetic field, Int. J. Nonlinear Mech. 40 (2005) 465-474.

[10] T. Hayat, Z. Abbas, Heat transfer analysis on the MHD flow of a second grade fluid in a channel with porous medium, Chaos Solitons Fractals 38 (2008) 556-567.

[11] M. Khan, Z. Abbas, T. Hayat, Analytic solution for flow of sisko fluid through a porous medium, Transp. Porous Media 71 (2008) 23-37.

[12] K. A. Yih, The effect of uniform suction/blowing on heat transfer of magnetohydrodynamic Hiemenz flow through porous media, Acta Mech. 130 (1998d) 147-158.

[13] A. Nakayama, H. Koyama, Free convection heat transfer over a non-isothermal body of arbitrary shape embedded in a fluid saturated porous media, ASME J. Heat Transfer 109 (1987) 125130.

[14] R. S. R. Gorla, A. Y. Bakier, L. Byrd, Effects of thermal dispersion and stratification on combined convection on a vertical surface embedded in a porous medium, Transp. Porous Media 25 (1996) 275-282.

[15] I. A. Hassanien, A. Y. Bakier, R. S. R. Gorla, Effect of thermal dispersion and stratification on non-darcy mixed convection from a vertical plate in a porous medium, Heat Mass Transfer 34 (1998) 209-212.

[16] M. A. Seddeek, Effects of non-Darcian on forced convection heat transfer over a flat plate in a porous medium with temper- ature dependent viscosity, Int. Commun. Heat Mass Transfer 32 (2005) 258-265.

[17] K. Parand, S. A. Hossayni, J. A. Rad, An operation matrix method based on bernstein polynomials for Riccati differential equation and Volterra population model, Applied Mathematical Modelling 40 (2016) 993-1011.

[18] S. Kazem, M. Shaban, J. A. Rad, Solution of the coupled Burgers equation based on operational matrices of d-dimensional orthogonal functions, Zeitschrift für Naturforschung A 67 (2012) 267-274.

[19] K. Parand, Y. Lotfi, J. A. Rad, An accurate numerical analysis of the laminar two-dimensional flow of an incompressible EyringPowell fluid over a linear stretching sheet, The European Physical Journal Plus 132 (2017) 397.

[20] J. A. Rad, K. Parand, Numerical pricing of American options under two stochastic factor models with jumps using a meshless local Petrov-Galerkin method, Applied Numerical Mathematics 115 (2017) 252-274.

[21] S. Liao, The proposed homotopy analysis technique for the solution of nonlinear problems, Ph.D. thesis, Shanghai, Jiao Tong University (1992).

[22] S. Liao, Beyond Perturbation: Introduction to the Homotopy Analysis Method, Chapman and Hall/CRC Press, Boca Raton, 2003.

[23] S. Liao, An explicit, totally analytic approximation of blasius viscous flow problems, I. J. NonLinear Mech. 34 (1999) 759778.

[24] S. Liao, On the analytic solution of magnetohydrodynamic flows of non-newtonian fluids over a stretching sheet, J. Fluid Mech. 488 (2003) 189-212.

[25] S. Liao, On the homotopy analysis method for nonlinear problems, Appl. Math. Comput. 147 (2004) 499-513.

[26] S. Liao, Comparison between the homotopy analysis method and homotopy perturbation method, Appl. Math. Comput. 169 (2005) 1186-1194.

[27] M. Sajid, T. Hayat, S. Asghar, Comparison between the HAM and HPM solutions of thin film flows of non-Newtonian fluids on a moving belt, Nonlinear Dynamics 50 (2007) 27-35.

[28] F. Allan, Derivation of the Adomian decomposition method using the homotopy analysis method, Appl. Math. Comput. 190 (2007) 6-14.

[29] S. Abbasbandy, The application of homotopy analysis method to nonlinear equations arising in heat transfer, Phys. Lett. A 360 (2006) 109-113.

[30] S. Abbasbandy, Approximate solution for the nonlinear model of diffusion and reaction in porous catalysts by means of the homotopy analysis method, Chem. Eng. J. 136 (2008) 144-150.

[31] F. M. Allan, Construction of analytic solution to chaotic dynamical systems using the homotopy analysis method, Chaos, Solitons and Fractals 39 (2009) 1744-1752.

[32] F. M. Allan, M. Syam, On the analytic solution of nonhomogeneous blasius problem, J. Comput. Appl. Math. 182 (2005) 362-371.

[33] T. Hayat, T. Javed, On analytic solution for generalized threedimensional MHD flow over a porous stretching sheet, Phys. Lett. A 370 (2007) 243-250.

[34] M. Sajid, A. Siddiqui, T. Hayat, Wire coating analysis using MHD Oldroyd 8-constant fluid, I. J. Eng. Sci. 45 (2007) 381392. 
[35] T. Hayat, M. Sajid, Analytic solution for axisymmetric flow and heat transfer of a second grade fluid past a stretching sheet, I. J. Heat Mass Tran. 50 (2007) 75-84.

[36] T. Hayat, N. Ahmed, M. Sajid, S. Asghar, On the MHD flow of a second grade fluid in a porous channel, Comput. Math. Appl. 54 (2007) 407-414.

[37] T. Hayat, M. Sajid, M. Ayub, A note on series solution for generalized Couette flow, Commun. Nonlinear. Sci. Numer. Simulat. 12 (2007) 1481-1487.

[38] X. Ran, Q. Zhu, Y. Li, An explicit series solution of the squeezing flow between two infinite plates by means of the homotopy analysis method, Commun. Nonlinear. Sci. Numer. Simulat. 14 (2009) 119-132.

[39] M. M. Rashidi, S. Dinarvand, Purely analytic approximate solutions for steady three-dimensional problem of condensation film on inclined rotating disk by homotopy analysis method, Nonlinear Anal. Real World Appl. 10 (2009) 2346-2356.

[40] M. M. Rashidi, G. Domairry, S. Dinarvand, Approximate solutions for the Burger and regularized long wave equations by means of the homotopy analysis method, Commun. Nonlinear. Sci. Numer. Simulat. 14 (2009) 708-717.

[41] Z. Ziabakhsh, G. Domairry, Solution of the laminar viscous flow in a semi-porous channel in the presence of a uniform magnetic field by using the homotopy analysis method, Commun. Nonlinear. Sci. Numer. Simulat. 14 (2009) 1284-1294.

[42] Z. Ziabakhsh, G. Domairry, H. Bararnia, H. Babazadeh, Analytical solution of flow and diffusion of chemically reactive species over a nonlinearly stretching sheet immersed in a porous medium, J. Taiwan Inst. Chem. Eng. 41 (2010) 22-28.

[43] Z. Ziabakhsh, G. Domairry, M. Mozaffari, M. Mahbobifar, Analytical solution of heat transfer over an unsteady stretching permeable surface with prescribed wall temperature, J. Taiwan Inst. Chem. Eng. 41 (2010) 169-177.

[44] T. Hayat, M. Qasim, Z. Abbas, Homotopy solution for the unsteady three-dimensional MHD flow and mass transfer in a porous space, Commun. Nonlinear. Sci. Numer. Simulat. 15 (2010) 2375-2387.

[45] A. Alizadeh-Pahlavan, K. Sadeghy, On the use of homotopy analysis method for solving unsteady MHD flow of Maxwellian fluids above impulsively stretching sheets, Commun. Nonlinear. Sci. Numer. Simulat. 14 (2009) 1355-1365.

[46] O. Abdulaziz, N. F. M. Noor, I. Hashim, Homotopy analysis method for fully developed MHD micropolar fluid flow between vertical porous plates, Int. J. Numer. Meth. Engng 78 (2009) 817-827.

[47] S. Abbasbandy, A. Shirzadi, A new application of the homotopy analysis method: Solving the Sturm-Liouville problems, Commun. Nonlinear. Sci. Numer. Simulat. 16 (2011) 112-126.

[48] J. A. Rad, S. M. Ghaderi, K. Parand, Numerical and analytical solution of gas flow through a Micro-Nano porous media: A comparison, J. Comput. Theor. Nanosci. (2011), in press.

[49] T. Hayat, R. Naz, S. Abbasbandy, Poiseuille flow of a third grade fluid in a porous medium, Trans. Porous Med. 87 (2011) 355-366.

[50] T. Hayat, Z. Iqbal, M. Sajid, K. Vajravelu, Heat transfer in pipe flow of a Johnson-Segalman fluid, I. Commun. Heat Mass Tran. 35 (2008) 1297-1301.

[51] F. Awawdeh, A. Adawi, Z. Mustafa, Solutions of the SIR models of epidemics using HAM, Chaos, Solitons and Fractals 42 (2009) 3047-3052.
[52] S. Abbasbandy, E. Babolian, M. Ashtiani, Numerical solution of the generalized Zakharov equation by homotopy analysis method, Commun. Nonlinear Sci. Numer. Simulat. 14 (2009) 4114-4121.

[53] A. Alomari, M. Noorani, R. Nazar, C. Li, Homotopy analysis method for solving fractional Lorenz system, Commun. Nonlinear. Sci. Numer. Simulat. 15 (2010) 1864-1872.

[54] L. A. Soltani, E. Shivanian, R. Ezzati, Shooting homotopy analysis method: A fast method to find multiple solutions of nonlinear boundary value problems arising in fluid mechanics, Engineering Computations 34 (2017) 471-498.

[55] L. A. Soltani, E. Shivanian, R. Ezzati, Convection-radiation heat transfer in solar heat exchangers filled with a porous medium: Exact and shooting homotopy analysis solution, Applied Thermal Engineering 103 (2016) 537-542.

[56] S. Abbasbandy, E. Shivanian, K. Vajravelu, Mathematical properties of $h$-curve in the frame work of the homotopy analysis method, Communications in Nonlinear Science and Numerical Simulation 16 (2011) 4268-4275.

[57] S. Abbasbandy, E. Magyari, E. Shivanian, The homotopy analysis method for multiple solutions of nonlinear boundary value problems, Communications in Nonlinear Science and Numerical Simulation 14 (2009) 3530-3536.

[58] S. Abbasbandy, E. Shivanian, Prediction of multiplicity of solutions of nonlinear boundary value problems: novel application of homotopy analysis method, Communications in Nonlinear Science and Numerical Simulation 15 (2010) 3830-3846.

[59] S. Abbasbandy, E. Shivanian, Predictor homotopy analysis method and its application to some nonlinear problems, Communications in Nonlinear Science and Numerical Simulation 16 (2011) 2456-2468.

[60] R. Ellahi, E. Shivanian, S. Abbasbandy, T. Hayat, Numerical study of magnetohydrodynamics generalized Couette flow of Eyring-Powell fluid with heat transfer and slip condition, International Journal of Numerical Methods for Heat and Fluid Flow 26 (2016) 1433-1445.

[61] S. Abbasbandy, E. Shivanian, A new analytical technique to solve Fredholm's integral equations, Numerical algorithms 56 (2011) 27-43.

[62] R. Ellahi, E. Shivanian, S. Abbasbandy, S. U. Rahman, T. Hayat, Analysis of steady flows in viscous fluid with heat/mass transfer and slip effects, International Journal of Heat and Mass Transfer 55 (2012) 6384-6390.

[63] E. Shivanian, S. Abbasbandy, Predictor homotopy analysis method: two points second order boundary value problems, Nonlinear Analysis: Real World Applications 15 (2014) 89-99.

[64] S. Kazem, M. Shaban, J. Rad, A new tau homotopy analysis method for mhd squeezing flow of second-grade fluid between two parallel disks, Applied and Computational Mathematics 16 (2017) 114-132.

[65] M. Shaban, S. Kazem, J. A. Rad, A modification of the homotopy analysis method based on Chebyshev operational matrices, Mathematical and Computer Modelling 57 (2013) 12271239.

[66] S. Abbasbandy, D. Modarrespoor, K. Parand, J. A. Rad, Analytical solution of the transpiration on the boundary layer flow and heat transfer over a vertical slender cylinder, Quaestiones Mathematicae 36 (2013) 353-380.

[67] K. Parand, S. Abbasbandy, S. Kazem, J. A. Rad, A novel application of radial basis functions for solving a model of first-order 
integro-ordinary differential equation, Commun. Nonlinear. Sci. Numer. Simulat. 16 (2011) 4250-4258.

[68] S. Kazem, J. A. Rad, K. Parand, S. Abbasbandy, A new method for solving steady flow of a third grade fluid in a porous half space based on radial basis functions, Zeitschrift für Naturforschung A 66 (2011) 591-598.

[69] S. Liao, Y. Tan, A general approach to obtain series solutions of nonlinear differential equations, Stud. Appl. Math. 119 (2007) 297.
[70] P. Hilton, An introduction to homotopy theory, Cambridge University Press, New York, 1953.

[71] S. Liao, An optimal homotopy-analysis approach for strongly nonlinear differential equations, Commun. Nonlinear. Sci. Numer. Simulat. 15 (2010) 2003-2016.

[72] H. A. Attia, Hiemenz flow through a porous medium of a nonNewtonian Rivlin-Ericksen fluid with heat transfer, Tamkang J. Sci. Eng. 12 (2009) 359-364. 